\title{
Non-detection of Dark Matter particles: A case for alternate theories of gravity
}

\author{
C Sivaram \\ Indian Institute of Astrophysics, Bangalore, 560 034, India \\ e-mail: sivaram@iiap.res.in
}

Kenath Arun

Christ Junior College, Bangalore, 560 029, India

e-mail: kenath.arun@cjc.christcollege.edu

\begin{abstract}
A Prasad
Center for Space Plasma \& Aeronomic Research, The University of Alabama in Huntsville, Huntsville, Alabama 35899

email: ap0162@uah.edu
\end{abstract}

\section{Louise Rebecca}

Christ Junior College, Bangalore - 560 029, India

e-mail: louise.rheanna@cjc.christcollege.edu

\begin{abstract}
While there is overwhelming evidence for dark matter (DM) in galaxies and galaxy clusters, all searches for DM particles have so far proved negative. It is not even clear whether only one particle is involved or a combination or particles, their masses not precisely predicted. This non-detectability raises the possible relevance of modified gravity theories - MOND, MONG, etc. Here we consider a specific modification of Newtonian gravity (MONG) which involves gravitational self-energy, leading to modified equations whose solutions imply flat rotation curves and limitations of sizes of clusters. The results are consistent with current observations including that involving large spirals. This modification could also explain the current Hubble tension. We also consider effects of dark energy (DE) in terms of a cosmological constant.
\end{abstract}


Over the past few decades there have been a plethora of sophisticated experiments involving massive sensitive detectors trying to catch faint traces of the elusive Dark Matter (DM) particles. But so far all of these efforts have been to no avail. Most of these detectors are designed to look for Weakly Interacting Massive Particles (WIMPS) which are much heavier than the proton (several $\mathrm{GeV}$ ), with there being no definite prediction for the masses of these heavy particles [1]. Another possible candidate is the axion, which is expected to have a much smaller mass $\left(10^{-3}-10^{-5} \mathrm{eV}\right)$. Here again there is no definite theoretical prediction for the axion mass. A recent work [2] has put limits on very light axion like particle. There has also been several astrophysical searches for DM particles.

So considering these negative results of all experiments so far, can we try to understand why we don't see them? While evidence for DM as such is overwhelming - otherwise galaxies and galaxy clusters would fly apart but for the additional gravity they provide - the question is what type of particles constitute them, or can there be alternate ideas to understand the enhanced gravity. Is only one type of DM particle involved or a combination? Theories do not predict what combination of particles or what type of particles will fix the ratio of DM to baryonic matter as about six. We suggest some reasons why we have not seen these elusive particles.

One such suggestion that has been made [3, 4] is that the DM particles (of favoured mass range) could form degenerate objects of a Neptune mass or less. The first DM clumps to form (as these particles do not couple to radiation) could form primordial planets at large redshifts. So this clumping of DM into objects of different masses would substantially reduce the flux of free DM particles, so that the number of expected events in detectors would be reduced, accounting for negative results so far. Even axions could clump [5].

Another possibility is that the DM particles could have much weaker cross-sections and their masses may not be in the range assumed. This would lead to non-detection. A more drastic conclusion would be that the particles in the predicted mass range, fluxes and coupling may not exist. They may be a different kind of particle, interacting only gravitationally with higher masses, the fluxes would be smaller and number of events less.

In the absence of detection of DM particles so far, it is natural to explore alternate possibilities such as modification of Newtonian gravity that could explain the galaxy rotation curves and motion of clusters. There have been recent approaches in this direction [6]. One such alternative picture, the Modification of Newtonian dynamics (MOND) was initially proposed as an alternative to account for the flat rotation curves of spiral galaxies, without invoking DM in the halo [7, 8]. 
The theory required an ad hoc introduction of a fundamental acceleration $a_{0} \approx$ $10^{-8} \mathrm{~cm} / \mathrm{s}^{2}$. When the acceleration approaches $a_{0}$, the Newtonian law giving the field strength is modified as:

$a=\frac{\left(G M a_{0}\right)^{1 / 2}}{r}$

where $a$ is the acceleration, $r$ is the radial distance, $M$ is the central mass. And this gives a constant velocity, i.e. flat rotation curve for the galaxies, with the constant velocity, at the galactic outskirts given by, $v_{c}=\left(G M a_{0}\right)^{1 / 4}$.

These results can also be arrived at by considering a minimum acceleration given by [9]:

$a_{\min }=\frac{G M}{r_{\max }^{2}}$

Here $r_{\max }$ is the radius of the structure corresponding to the minimum acceleration and it sets the limit for the size of large scale structures, which follows from equation (2) as, $r_{\max }=$ $\left(\frac{G M}{a_{\min }}\right)^{1 / 2}$, hence we get,

$v_{c}=\left(G M a_{\min }\right)^{1 / 4}$

This velocity is independent of $r$ beyond $r_{\max }$, which is consistent with observation. For Milky Way, this constant velocity (equation (3)) $\sim 300 \mathrm{~km} / \mathrm{s}$, same order as that observed. For clusters of galaxies, such as $\operatorname{Virgo}\left(M \approx 1.25 \times 10^{15} M_{\odot}\right)$ and $\operatorname{Coma}\left(M \approx 7 \times 10^{14} M_{\odot}\right)$ clusters, the velocity (from equation (3)) is $\sim 1500 \mathrm{~km} / \mathrm{s}$ which is again in accordance with what is observed. The constraints on the size of large scale structures such as galaxies, clusters and super clusters, i.e. $r_{\max }=\left(\frac{G M}{a_{\min }}\right)^{1 / 2}$, closely matches with observations (table 1) [10].

Table 1: Observed and calculated sizes of clusters and superclusters

\begin{tabular}{|l|c|c|}
\hline Large scale structure & $\boldsymbol{r}_{\text {lim }}($ in cm) & $\boldsymbol{r}_{\text {obs }}($ in cm) \\
\hline Virgo Cluster & $2.28 \times 10^{24}$ & $7.09 \times 10^{24}$ \\
\hline Coma Cluster & $2.16 \times 10^{25}$ & $9.46 \times 10^{24}$ \\
\hline Omega Centauri & $1.6 \times 10^{18}$ & $8.13 \times 10^{19}$ \\
\hline Saraswati Supercluster & $1.15 \times 10^{25}$ & $2 \times 10^{26}$ \\
\hline Laniakea Supercluster & $2.58 \times 10^{25}$ & $2.36 \times 10^{26}$ \\
\hline Horologium Supercluster & $2.5 \times 10^{25}$ & $5 \times 10^{26}$ \\
\hline Corona Borealis Supercluster & $7.47 \times 10^{25}$ & $3.1 \times 10^{26}$ \\
\hline
\end{tabular}


It was also discussed in recent papers that the requirement that the attractive gravitational binding self-energy density of large scale structures (such as galaxies, clusters, superclusters, etc.) should at least be equal to the background repulsive DE (cosmological constant $\Lambda$ ) density implies a mass-radius relation of the type [11-13]:

$\frac{M}{r^{2}}=\frac{c^{2}}{G} \sqrt{\Lambda} \approx 1 \mathrm{~g} / \mathrm{cm}^{2}$

for the observed value of $\Lambda \sim 10^{-56} \mathrm{~cm}^{-2}$. Here $M$ and $r$ corresponds to mass and radius of these structures. This relation holds true for primeval galaxies as well as those at present epoch [14]. This relation can also be obtained by rearranging equation (2), i.e., $\frac{M}{r_{\max }^{2}}=\frac{a_{\min }}{G} \approx$ $1 \mathrm{~g} / \mathrm{cm}^{2}$, where the minimum acceleration is $a_{\min } \sim 10^{-8} \mathrm{~cm} / \mathrm{s}^{2}$.

The flat rotation curves can also be explained by considering Modifications of Newtonian Gravity (MONG). By adding a gravitational self-energy term to the Poisson's equation we get,

$\nabla^{2} \phi+K(\nabla \phi)^{2}=4 \pi G \rho$

where $\phi\left(\sim \frac{G M}{r}\right)$ is the gravitational potential and the constant $K \sim G^{2} / c^{2}$. The gravitational self-energy density is given by $K(\nabla \phi)^{2}$, and also contributes to the gravitational field along with the matter density $\rho$. For small values of the density $\rho$, (for e.g. at the outskirts of galaxies) we have,

$\nabla^{2} \phi+K(\nabla \phi)^{2}=0$

The solution of this equation yields, $\phi=K^{\prime} \ln \frac{r}{r_{\max }}$

where $K^{\prime}=\frac{G M}{r_{\max }}$ is a constant. This gives the force of the form,

$F=\frac{K^{\prime \prime}}{r}$

where $K^{\prime \prime}=\left(G M a_{\min }\right)^{1 / 2}$, is again a constant. The balance of centripetal force and gravitational force then gives, $v^{2} / r=K^{\prime \prime} / r$.

This then implies the independence of $v$ on $r$ (i.e. flat rotation curve, $v^{2}=K$ ", which is a constant) for larger distances from the centre of the galaxy (i.e. for $r>r_{\text {max }}$ ). Including both gravitational self-energy and DE densities, the Poisson's equation now takes the form,

$\nabla^{2} \phi+K(\nabla \phi)^{2}-\Lambda c^{2}=0$

The general solution (for the potential $\phi$ ) can be written as:

$\phi=\frac{G M}{r}+K^{\prime} \ln \frac{r}{r_{\max }}+\Lambda r^{2} c^{2}$ 
We now make use of this general solution for different regimes of interest in the galaxy structure. Where matter density dominates, i.e. $r<r_{\max }$, we have $\phi \approx \frac{G M}{r}$ (solution of $\nabla^{2} \phi=$ $4 \pi G \rho$ ) which gives a velocity varying linearly with distance. For $r>r_{\max },(\nabla \phi)^{2}$ term dominates, and $\phi$ goes as $K^{\prime} \ln \frac{r}{r_{\max }}$, (giving a constant velocity) accounting for DM (solution of $\nabla^{2} \phi+K(\nabla \phi)^{2}=0$ ). For $r \gg r_{\text {max }}$, $\phi$ goes as $\Lambda r^{2} c^{2}$, DE dominates (i.e. the cosmological constant term).

In the case of the Milky Way, the velocity flattens out beyond $\sim 2 \mathrm{kpc}$, which is what is obtained from the above results (figure 1). Other galaxies also show similar typical rotation curves, with MONG matching with observation [15].

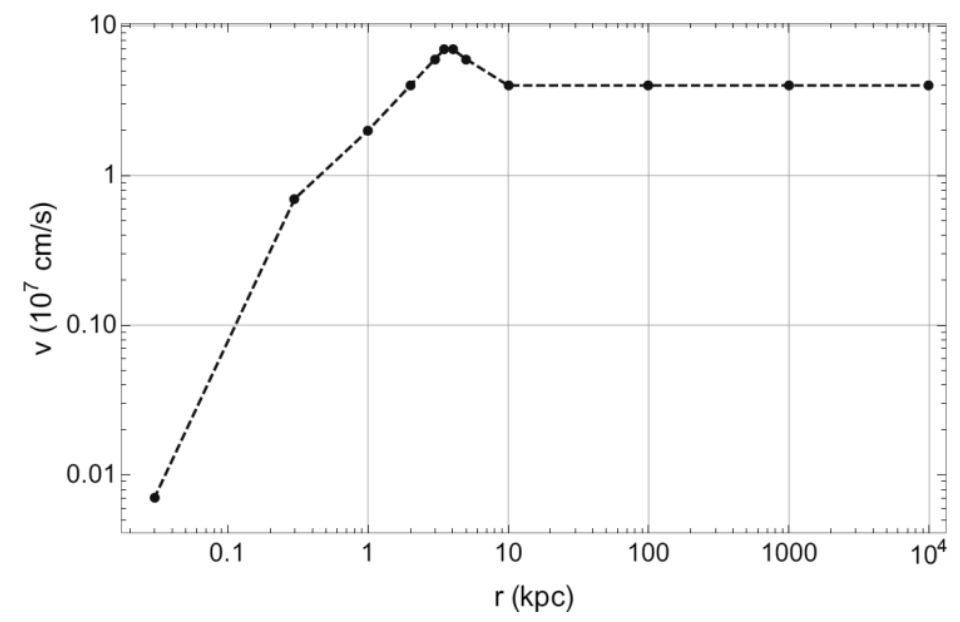

Figure 1: Galaxy rotation curve (for Milky Way) from modified Newtonian gravity

In this connection, the extra term in the Poisson equation given by MONG, i.e. equation (9) and its solution given by equation (10) could have interesting consequences for current observations of super-spirals [16], wherein their large extent (450,000 light-years) is associated with large rotation velocities of up to $\sim 450 \mathrm{~km} / \mathrm{s}$ at their periphery. Conventionally such large velocity would imply a large amount of DM, i.e. about $\sim 10^{13} M_{\odot}$. However, our extra term would give a velocity given by:

$v=\left(G M a_{\min }\right)^{1 / 4}\left(\ln \frac{r}{r_{\max }}\right)^{1 / 2}$

Where $r_{\max }$ corresponds to the radius at which acceleration approaches $a_{\min }$. With $r_{\text {max }}=20 \mathrm{kpc}$ and super spiral extant $r=r_{S S} \approx 200 \mathrm{kpc}$, this would give velocities $\sim 450 \mathrm{~km} / \mathrm{s}$. In other words, the logarithmic term makes gravity stronger above $r_{\max }$ (i.e., potential going as $\ln r$, instead of $1 / r$ ), so that we do not need such colossal amounts of DM. This also implies a logarithmic correction to the Tully-Fisher relation. 
The usual Friedmann equation now gets modified to:

$\frac{\dot{R}^{2}}{R^{2}}=\frac{8 \pi G \rho}{3}+\left(G M a_{0}\right)^{1 / 2} \ln \frac{R}{R_{\max }}$

( $R$ is the scale factor)

The second term can be seen as a modification in potential energy due to gravitational self-energy density in the usual balance between kinetic and potential energy terms, $\frac{8 \pi G \rho}{3}$ (in the expanding Universe), i.e. the usual Newtonian analogue agreeing with the GR result.

With $a_{0}=10^{-8} \mathrm{~cm} / \mathrm{s}^{2}$, and the Universe having expanded at present to $R>10^{28} \mathrm{~cm}$ the modified term will also contribute. With $R \approx 2 \times 10^{28} \mathrm{~cm}, \rho \approx 10^{-29} \mathrm{~g} / \mathrm{cc}$, and the mass of the Universe, $M=2 \pi^{2} R^{3} \rho \approx 10^{56} \mathrm{~g}$, the usual first term is $\approx 10^{21}$, whereas the second term $\approx 10^{20}$. This suggests that this extra term now manifesting itself would cause an increase of the expansion rate, i.e. a change in Hubble constant $\left(\dot{R}^{2} / R^{2}\right)$ by $\sim 5 \%$. This could perhaps account for the faster expansion rate seen at the present epoch. Hence this modification of the gravitational field can provide an alternate explanation for the discrepancy in the value of the Hubble constant as implied by Planck observations of the CMBR in the early Universe and that deduced from other distance indicators in the present epoch [17-19].

Hence we see that the specific modification of Newtonian gravity, involving gravitational self-energy leads to modified equations and the solutions imply flat rotation curves and limits the sizes of clusters. This modification can also account for current observations involving super-spiral galaxies and can account for the Hubble tension.

\section{References:}

[1] Arun, K, Gudennavar, S B and Sivaram, C (2017) Adv. Space Res., 60, 166

[2] Reynolds, C S et al. (2020) Astrophys. J., 뭉, 59

[3] Sivaram, C, Arun, K (2011) Open Astron. J., 4, 57

[4] Sivaram, C, Arun, K and Kiren, O V (2019) Earth, Moon and Planets, 122, 115

[5] Hogan, C J and Rees M J (1988) Phys. Lett. B, 205, 228

[6] Sivaram, C, Arun, K and Rebecca, L (2020) J. Astrophys. Astron., 41, 4

[7] Milgrom, M (1983) Astrophys. J., 270, 365

[8] Milgrom, M (1983) Astrophys. J., 270, 371

[9] de Sabbata, V and Sivaram, C (1993) Found. Phys. Lett., $\underline{6}, 561$

[10] Rebecca, L, Arun, K and Sivaram, C (2018) Astrophys. Space Sci., $\underline{363}, 149$

[11] Sivaram, C and Arun, K (2012) Open Astron. J., 5, 7 
[12] Sivaram, C and Arun, K (2013) Astrophys. Space Sci., 348, 217

[13] Sivaram, C, Arun, K and Kiren, O V (2013) Open Astron. J., ㅁ, 90

[14] Sivaram, C, Arun, K and Rebecca, L (2020) J. Astrophys. Astron., 41, 4

[15] Rebecca, L, Arun, K and Sivaram, C (2019) Indian J. Phys. https://doi.org/10.1007/s12648 $-019-01591-8$

[16] Ogle, P M et al. (2019) Astrophys. J. Lett., $\underline{884}$, L11

[17] Aghanim, $\mathrm{N}$ et al. (2018) arXiv:1807.06209v2 [astro-ph.CO]

[18] Riess, A et al. (2019) Astrophys. J., $\underline{876}, 85$

[19] Freedman, W. L. et al. (2019) Astrophys. J., $\underline{882}, 34$ 